\title{
Smart curiosity sink node prediction mining algorithm for path optimization in wireless sensor network
}

\author{
Dr. A. Kannagi ${ }^{{ }^{*}}$ \\ ${ }^{l}$ Professor, Department of Computer Science and Engineering, Malla Reddy College of Engineering, Tamilnadu, India. \\ *Corresponding author E-mail:kannagianbu@gmail.com
}

\begin{abstract}
:
As of late, data administration and preparing for WSNs have turned into a subject of dynamic research in a few fields of software engineering, for example, the dispersed frameworks, the database frameworks, and the data mining. Dealing with the extensive sums of information and efficiently using this information in improved path optimization has become increasingly challenging. In this paper, we will demonstrate how sink node predicted and integrated for path relationships and patterns in linked data, i.e., the interdependencies between data items at the lowest elemental level. The problem of path optimization has been approached using various techniques. The path selection plays the vital role in achieving the quality of service parameter and secure communication. Considering multiple routing, the security can be enforced with various strategies. Using few parameters namely the congestion, delay and hop count would support improve the performance of the network as well as lifetime. With the motivation, an efficient smart curiosity sink node prediction mining algorithm has been presented in this paper. First, both the source and destination nodes maintain information about the routes and network conditions. Based on that a single efficient path has been selected for data transmission. On the other side, the receiver verifies the path being followed, the route available and their conditions. Using this information the delay approximation is performed to decide the legitimate of the path being selected and the traffic incur in the way. The proposed method identifies several network threats and detects the presence of the node in the route. The proposed plan improves the performance of mining data efficiency as well as increases the throughput.
\end{abstract}

Keywords: Sink node, path optimization, mining, route estimation, WSN.

\section{Introduction}

Machine learning from sensor data has gotten a lot of consideration by the data mining group. Distinctive methodologies concentrating on path optimization, sink node recognizable proof, regular examples, following examples, and characterization have been efficiently utilized on sensor data. Be that as it may, the outline and sending of sensor networks make one of a kind research challenges because of their massive size irregular and unsafe execution, lossy imparting condition, restricted power supply, and high disappointment rate.

These difficulties make conventional mining strategies inapplicable because customarily mining is brought together and computationally costly.Wireless sensor networks have attracted significant attention in many applications domains, such as habitat monitoring, object tracking, environment monitoring, military, disaster management, as well as smart environments. In these applications, real-time and reliable control is an essential requirement. These applications yield the massive volume of dynamic, geographically distributed and heterogeneous data. This crude data, if productively broke down and changed to usable data through data mining, can encourage mechanized or humanincited strategic/key choice. Thusly, it is fundamental to create systems to dig the sensor data for examples to make intelligent decisions promptly.

Subsequently, new calculations have been made, and a portion of the data mining calculations have been altered to deal with the data produced by sensor networks. Plenty of information disclosure procedures.Since data mining is an expansive demonstrate and can be connected to any area data, more broad overviews on data mining systems, where creators analyzed the machine-learning and data mining methods for path optimization amid data move in the network.

Since the order of data mining procedures in this evaluation depends on path design mining, sink node, and it follows, there are adequate of examinations accessible on every one of these methods. The smart curiosity sink node prediction mining procedures focus on design and management of network instead of data disclosure. The sink node expectation mining systems strategies over data stream are given, where the source analyzed conventional characterization methods over data streams.

In any case, inspected data mining methods that importance on path optimization and examination from WSNs data. In correlation with the calculations supposed above and approaches uncommonly intended for WSNs, not just promoting an alternate order, assessment, and exchange on different spaces yet also exhibiting several decisions of a solution. We inspected how data mining calculations will be used to influence the sensor to network applications wise. In this work comprises of sink node forecast of data mining systems for WSNs.

\section{Related works}

A choice tree is a straightforward and intense type of data examination which enables us to foresee, clarify, depict, or arrange an objective. A choice tree comprises of two sorts of nodes; nonterminal nodes and terminal nodes [1]. A non-terminal node signifies a condition in view of an element. This condition parts data records in view of their qualities. A terminal node, additionally 
it is known as a leaf node, doesn't have a term because it is used to make a decision.

Data mining obtained from the data mining techniques and trained through mathematical models were validated with corresponding surveillance data. An basic class of these frameworks in body sensor systems (BSNs) here lightweight implanted processors and correspondence frameworks are firmly combined with the human body [2].

Upgraded Mining of High Dimensional Data utilizing Efficient Clustering approach called regulated property bunching calculation is proposed to enhance the precision and check the likelihood of the examples. In this strategy, speedier recovery of pertinent data is made more productive and exact [3]. By utilizing this technique, clients can get exact outcomes and immaterial data misfortune. This strategy shows comes about in light of the high likelihood thickness in this way giving security to data and decreasing fthe dimensionality of the data.

BSNs can give specialists, mind suppliers and clinicians access to immensely significant data extricated from data that are gathered. To address this issue, a data mining approach motivated by the involvement in the regions of content and characteristic dialect handling [4]. Speak to sensor readings with a succession of characters, called movement transcripts. Transcripts lessen the multifaceted nature of the data essentially while keeping up morphological and auxiliary properties of the physiological signs.Enabling location-aware quality-controlled access in wireless networks framework of provisioning service differentiation and fairness according to the per-location target load. In the proposed structure, the load information is feedback to traffic senders and used to adjust their sending rate [5], so that per-location weight does not deviate from a given per-location share of wireless link capacity and lays only tolerable traffic on the network in cooperation with other locations.

The highlights of the preparation tests were separated to develop the choice table; the harsh set hypothesis was connected to decrease the choice table. At long last, the decreased choice credits were utilized to build neural system order data [6]. Assembled data mining calculation can be incorporated into every sensor organize node. The issues need to tackle are changing as the time past, to plan a framework which can be working longer, it must be versatile.

The course of data mining research, at that point portrays the examination on remote sensor organize data mining, plans the Service Oriented Sensor Network Data Mining System, and incorporates three calculations: appropriated k-implies, multi-target following. In view of the quick data arriving speed and the monstrous size of data set in stream show [7], novel one-pass calculations are conceived to help data accumulation on request.

Finish movement observing and programmed fall recognition for indoor and open air utilize. The framework is made out of a portable module worn by the client and call focus spare and investigations the data [8]. Movement observing is performed utilizing neural systems. Remote accelerometer sensor module and calculation for determining fall identification. The fall recognition calculation recognizes wearer's stance, movement, and fall.

Data administration is the errand of gathering data from sensors, putting away data in the system, and productively convey data to the clients (the sinks) [9]. The comprehension of data administration methods executed in today sensor systems is required before tending to abnormal state data mining, design acknowledgment, and dispersed speculation development.

Constant worldwide situating has been supporting limitation and following in mining for a considerable length of time [10]. An earthly beneficiary can decide its situation from a known code succession originating from at least four satellites of known area, utilizing the synchronization-decided time of flight and the season of transmission and satellite area, which are additionally incorporated into each flag.

\section{Materials and methods}

The efficient smart curiosity sink node prediction mining algorithm maintains traces about sink node present in each transfer; the process has the number of stages Source Discovery based on Delay Approximation, ID-based Sink Detection, Path Setup for Secure Communication and Distributed Authentication.

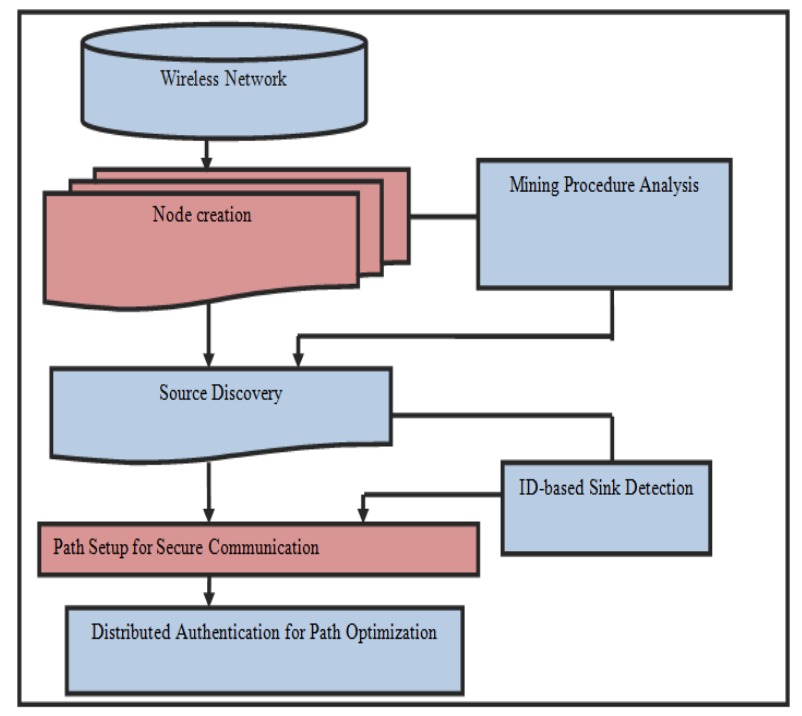

Figure 3.1: Work flow of proposed method

The Figure 3.1, shows the proposed work efficient smart curiosity sink node prediction mining algorithm for path prediction in wireless sensor network and its functional components. We discuss each of the functional components in detail in this given section.

\subsection{Source discovery based on delay approximation}

The source discovery is the initial process of path optimization, and the method identifies the list of sources which possess the data. The nodes have limited delay, and the way determines the list of nodes which has data to be collected using the neighbor nodes. Initially, the node floods the message and receives the replies from all the nodes of the network. From the response being received the node extracts the list of nodes has information to be collected. By accepting all replies with the message about the data content or to identify the correct sources in the network. Once all the response is received, then the method extracts the list of source nodes, and to collects the delay information from all sources in the network.

\section{Algorithm}

Input: Number of Nodes NN

Output: Source Discovery SD

Start:

Step1: Read node $n$

Step2: Extract Node history Nh

Step3: Send message to all neighbors nodes

Step4: If Nh> Payload-Threshold then

Neighbor's nodes receive the floods

Step5: Else

Extracts the list of source nodes

Step6: End

Stop:

\subsection{ID based sink detection}

ID based sink detection uses the node id with mining administration to give the conclusion to end confirmation. In the system, each node 
keeps id and its relating record esteem, in view of ID and its list esteem the sink will be picked rapidly. Way advancement plot depends on course development parcel from the source node and a course answer bundle from the goal node. At whatever point a source node wants to attempt secure correspondence with the goal node, the source node shapes its sink node and sends to the data in the network and waits for a reply message from sink node. Based on sink node reply message the next step carry on. The list of Sources belongs to the node has been identified as follows:

Node Id set NIS $=\int_{i=1}^{\text {Size }(\text { Atr })} \sum$ Atr $(i)$. Source $==$ Source

The list of node id belongs to the transmission route has been identified as follows:

$$
\text { Route set Rs }=\int_{i=1}^{\text {size }(\text { Atr })} \sum \operatorname{Atr}(i) . \text { Route } \nexists R s
$$

The common hostnames can be computed as follows:

Common hosts $=\int \sum$ Host $\in \forall($ Routes of $r s)$

According to this, the routing factor can be estimated as follows:

$$
\text { Route factor } \mathrm{Rf}=\int_{i=1}^{\operatorname{size}(M t)} \frac{\sum M t(i) \in \forall C H N}{\operatorname{size}(M t r)}
$$

Similar to that the number of the malicious packet in the route can be computed as follows:

$$
\mathrm{Nr}==\int_{i=1}^{\text {size }(M t)} \sum M t(i) \text {. Route }==\text { Route }
$$

Using all these measures, the Sink can be computed as follows:

$$
\text { Sink }=\mathrm{Rf} \times \mathrm{Nr}
$$

The computed sink node has been used to perform path setup. The algorithm computes the sink detection factor for each route and identifies the moderate hops.

\section{Algorithm}

Input: Source Discovery Sd

Output: Sink

Start:

Step1:

Step2:

Read Source node Id

Step3:

Compute Delay

Step4:

Compute Transmission Weight

Step5:

Weights > Threshold

Step6: If Route Weight> Threshold

Sink node is detected

Step7: $\quad$ Else

Node id will be checked

Step8: $\quad$ End

Stop:

\subsection{Path setup for secure communication}

The path setup is essential one in the network after the sink node reply the source node require not have gone along the way with minimal number of jumps; the minimum bounce way may have a higher deferral, either honestly or malevolently showed. For this situation, be that as it may, a no congested, non-minimum jump way is probably going to be liked to a safe correspondence on account of the diminishment in delay. Since sink don't contain a jump check or particular recorded source course, and on the grounds that messages are encoded at each bounce, malignant nodes have no chance to divert movement with the adventures. After way setup, the goal unicasts an answer bundle back along the invert way to the source. In the middle of the node, check its encoded data and pass the reaction message to its next node the other way.

Algorithm:

Input: Sink node id SId,

Output: Correct path

Start:

Step1: $\quad$ Read Sink node

Step2: Read Node ID

Step3: Identify the ID of the source Sid

Sink node $\mathrm{Sn}=\int_{i=1}^{\operatorname{size}(S n)} \sum \operatorname{Sn}(i) . S I d=$

Sources. SId
Split the sink node into different time window

$\mathrm{Tw}=\int_{i=1}^{\operatorname{size}(T w)} \sum \operatorname{Sn}(i)$. Time

Step4: Now for each time window

Compute path $\mathrm{Cp}=\frac{\sum \operatorname{Sn} \in T w(i)}{\operatorname{Size}(s s)}$

Stop:

\subsection{Distributed authentication}

It's the last stage for path optimization after all the path setup source data will be transferred into a destination using sink node. That time used multiple intermediate nodes in the network, the source node distributed in all intermediate nodes during communication.

In the distributed authenticated secure communication scheme, individual nodes of relatively high safety among the sink nodes consisting of the network were set as source Nodes, from which the process of issuing a node ID was started and distributed.

\section{Result and discussion}

Efficient smart curiosity sink node prediction mining algorithm has been implemented in network simulator NS 2.28 version under various scenarios. The behavior of the suggested approaches is analyzed with a variety of simulation parameters. A node can straightforwardly impart to the nodes that exist in its correspondence extend.

In the event that a node needs to speak with a node that isn't straightforwardly inside its correspondence go, it utilizes transitional nodes as switches.

In a node from an area to another area can be empowered utilizing the catchphrase "setdest" in Tool Command Language (TCL) content. Nodes are designed with the componentsof the channel, organizing interface, radio spread model, Medium Access Control (MAC) convention, adhoc steering convention, interface line, connect layer, geology question, and recieving wire write.

Nodes in the system have been exchanged utilizing correspondence show.

The developments of versatile nodes are kept to a zone of $500 \mathrm{~m} \square 500 \mathrm{~m}$ with the interruption time of $3 \mathrm{~s}$. Data transmission is built up between nodes utilizing UDP specialist and CBR movement. These halfway switches forward the parcels produced by different nodes to their goal. Our proposed system (Smart Curiosity Sink Node Prediction (SCSNP)) is compared with three existing systems; they are Model-based clustering Algorithm (MBCA) and K-Partitioned Smallest Distance Tree (k-PSDT)

\subsection{Throughput Ratio}

The throughput proportion characterizes the rate of data parcels got at a goal as per the quantity of bundles produced by the source node for a specified period.

Throughput $=$ Received data $* 8 /$ Data transmission period

Table 4.1 shows the throughput ratio analysis of the proposed system with the existing systems.

Table 4.1 Throughput Ratio

\begin{tabular}{|c|c|c|c|}
\hline Number of nodes & $\begin{array}{c}\text { MBCA } \\
\text { in \% }\end{array}$ & $\begin{array}{c}\text { k-PSDT } \\
\text { in } \%\end{array}$ & $\begin{array}{c}\text { SCSNP } \\
\text { in \% }\end{array}$ \\
\hline 20 & 3.6 & 5.9 & 20.2 \\
\hline 40 & 18.6 & 19.2 & 23.5 \\
\hline 60 & 39.2 & 42.7 & 49.2 \\
\hline 80 & 59.3 & 65.1 & 75.2 \\
\hline 100 & 65.1 & 71.6 & 91.6 \\
\hline
\end{tabular}

Figure 4.1 shows the comparative analysis of throughput ratio of the proposed scheme with the existing, based on the values is given below. 


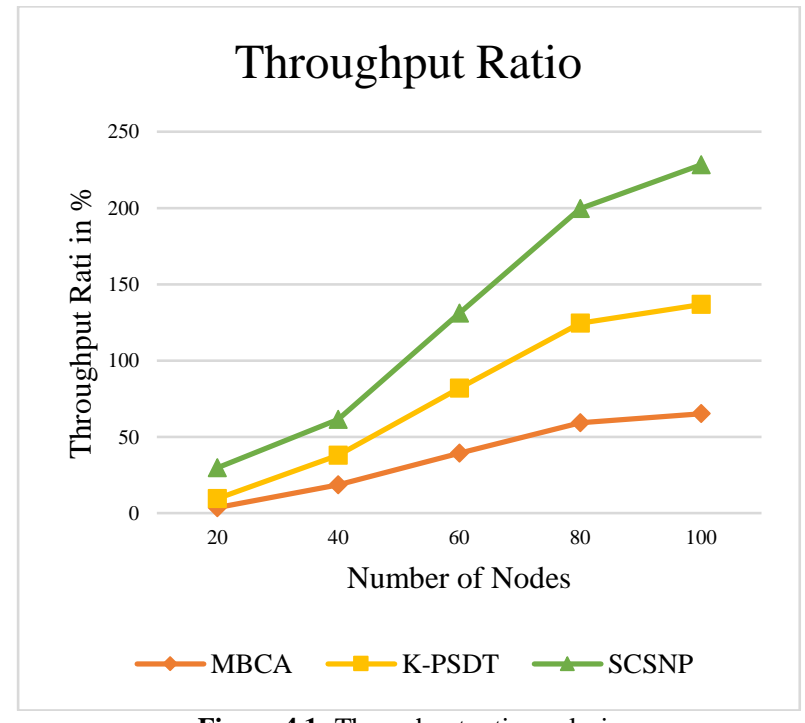

Figure 4.1: Throughput ratio analysis

\subsection{Analysis of packet delivery ratio}

Packet Delivery Ratio (PDR) is used to evaluate the quality of the network.

It defines the ratio between the packets received by the destination and the packets generated by the source.

It can be obtained by using awk script, which produces the trace file and the result.

PDR $=$ Received packets $/$ Generated packets $* 100$

Table 4.2: Packet Delivery Ratio Comparative Analysis

\begin{tabular}{|c|c|c|c|}
\hline Number of nodes & $\begin{array}{c}\text { MBCA } \\
\text { in \% }\end{array}$ & $\begin{array}{c}\text { k-PSDT } \\
\text { in \% }\end{array}$ & $\begin{array}{c}\text { SCSNP } \\
\text { in \% }\end{array}$ \\
\hline 20 & 0 & 0 & 0 \\
\hline 40 & 29.75 & 39.58 & 47.65 \\
\hline 60 & 33.65 & 43.65 & 49.79 \\
\hline 80 & 49.28 & 61.98 & 72.89 \\
\hline 100 & 69.78 & 72.45 & 89.65 \\
\hline
\end{tabular}

Figure 4.2 gives the comparative analysis output of the proposed scheme with the existing approaches. The analysis depicts the following:

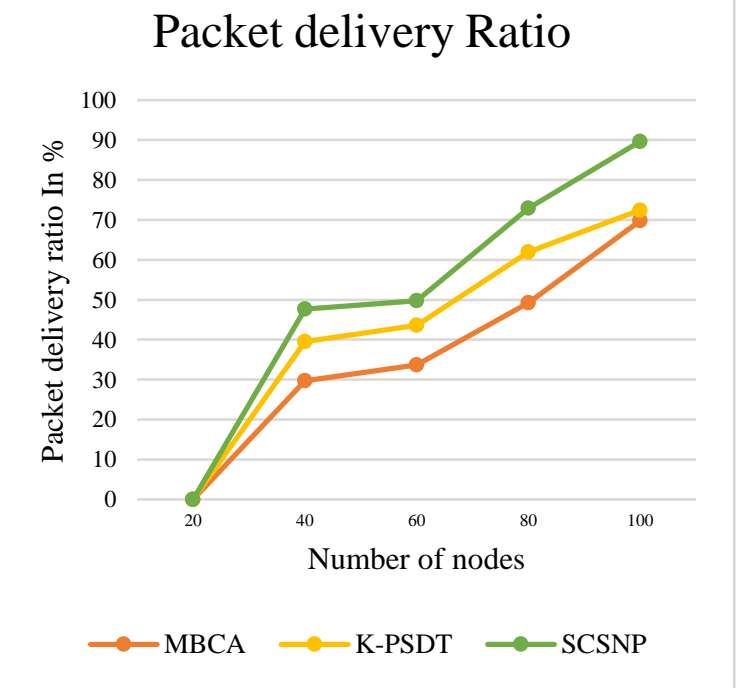

Figure 4.2 Packet Delivery Ratio Comparative Analysis

\subsection{Mining efficiency ratio}

The mining efficiency ratio is used to evaluate the quality of the network provided by the routing scheme. The simulation results of efficiency ratio are given in Table 4.3.
Table 4.3: Mining Efficiency Performance Analysis with Existing and Proposed Systems

\begin{tabular}{|l|l|l|l|}
\hline Number of Nodes & $\begin{array}{l}\text { MBCA } \\
\text { in } \%\end{array}$ & $\begin{array}{l}\text { k-PSDT } \\
\text { in } \%\end{array}$ & $\begin{array}{l}\text { SCSNP } \\
\text { in } \%\end{array}$ \\
\hline 20 & 19.72 & 21.95 & 23.65 \\
\hline 40 & 26.98 & 32.58 & 39.85 \\
\hline 60 & 39.68 & 49.85 & 55.68 \\
\hline 80 & 51.85 & 59.68 & 62.98 \\
\hline 100 & 73.65 & 82.15 & 91.58 \\
\hline
\end{tabular}

Figure 4.3 depicts the performance of the proposed system SCSNP with other systems. The proposed scheme outperforms the existingone regarding ratio.

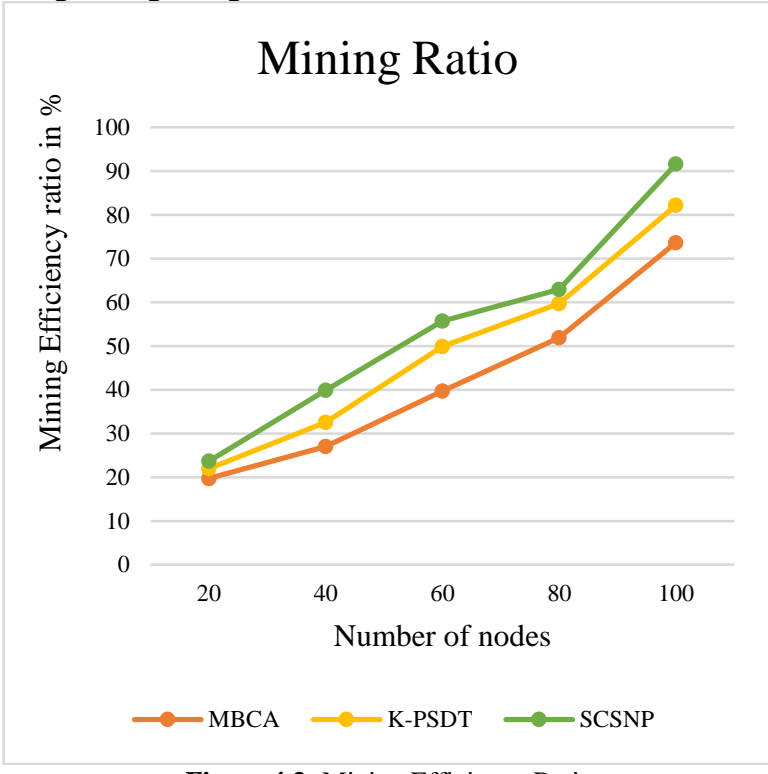

Figure 4.3: Mining Efficiency Ratio

\subsection{Analysis of end to end delay}

The time which is taken by the source node to deliver the data successfully to the destination is called as an end to end delay. It is the difference between the time at which packets generated by the sender and the time the packets received by the receiver and it can be obtained from the trace file.

End to end delay $=$ Received time - Send time

Table 4.4 shows the end to end delay analysis of the proposed system with the existing systems.

Table 4.4: End to End Delay

\begin{tabular}{|c|c|c|c|}
\hline Rate of Sending Packets/sec & MBCA & k-PSDT & SCSNP \\
\hline 10 & 1.23 & 0.86 & 0.59 \\
\hline 20 & 5.8689 & 4.5689 & 4.3658 \\
\hline 30 & 4.9658 & 3.8698 & 2.9875 \\
\hline 40 & 7.5854 & 7.1025 & 6.9358 \\
\hline 50 & 7.6549 & 6.5698 & 5.9868 \\
\hline
\end{tabular}

Figure 4.4 shows the end to end analysis of the proposed scheme with the existing systems. The delay calculated based on the rate of sending packets in particular seconds. Be that as it may, the slight variety is there. The proposed design prompts just endured delay in the system, despite the fact that the packets/sec. If the destination moves closer to the source node, the suspension will be decreased. Otherwise, the delay will be increased. 


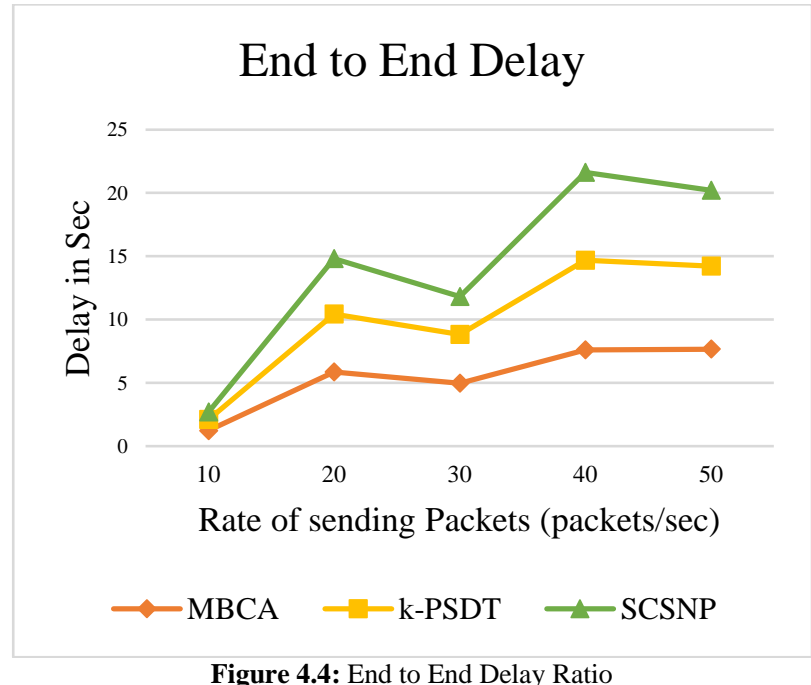

From Figure 4.4, it is understood that the proposed SCSNP has reduced its end to end delay with the rate of sending packets.

\section{5. conclusion}

The smart curiosity sink node prediction mining algorithm is performed based on the computed value of the sink detection. Here the path optimization of every node is evaluated and calculates the sink of the node. Using this sink node, the path section performed by analyzing, whether, the sink node is available or not. The proposed method after evaluation increase the throughput ratio up to $91.6 \%$, and produce the mining efficiency up to $91.58 \%$ in network. Also, our proposed model gives higher delivery ratio compared to all other existing methods in the network.

\section{References}

[1] Li L, Li X, Lu Z, Lloret J \& Song H, "Sequential Behavior Pattern Discovery with Frequent Episode Mining and Wireless Sensor Network”, IEEE Communications Magazine, Vol.55, No.6, (2017), pp.205-211.

[2] Kim H, Kim HS, Lee SK, Park EC \& Park KJ, "Enabling locationaware quality-controlled access in wireless networks", EURASIP Journal on Wireless Communications and Networking, (2011).

[3] Rao BT \& Reddy BS, "Mining of High Dimensional Data using Efficient Feature Subset Selection Clustering Algorithm (WEKA)", International Journal of Computer Applications, Vol.107, No.6, (2014).

[4] Loseu V, Ghasemzadeh H \& Jafari R, “A Mining Technique Using \$ N \$ n-Grams and Motion Transcripts for Body Sensor Network Data Repository", Proceedings of the IEEE, Vol.100, No.1, (2012), pp.107-121.

[5] Phung ND, Gaber MM \& Rohm U, "Resource-aware online data mining in wireless sensor networks", IEEE Symposium on Computational Intelligence and Data Mining, (2007), pp. 139-146.

[6] Yuehua H, Shuang X \& Huajian, W, "Study on distributed data mining model in Wireless Sensor Networks", IEEE International Conference on Intelligent Computing and Integrated Systems (ICISS), (2010), pp. 866-869.

[7] Ye XG, He YH \& Wu L, "Service Oriented Distributed Data Mining System in Sensor Network", IEEE Third International Conference on Information and Computing (ICIC), (2010), pp.225-228.

[8] Wang B, Wang T \& Mikou N, "An Efficient Data Streams Mining Method for Wireless Sensor Network's Data Aggregation", IEEE First International Workshop on Education Technology and Computer Science, Vol.3, (2009), pp.1016-1020.

[9] Ren SQ \& Park JS, "Density mining based resilient data aggregation for wireless sensor network", IEEE Fourth International Conference on Networked Computing and Advanced Information Management, Vol.1, (2008), pp.261-266.

[10] Perolle G., Fraisse P, Mavros M., \& Etxeberria I, “Automatic fall detection and activity monitoring for elderly", Proceedings of Medetel., (2006), pp. 65-70
[11] Lee Y, Kim J, Son M \& Lee, M, "Implementation of accelerometer sensor module and fall detection monitoring system based on wireless sensor network", IEEE 29th Annual International Conference of the Engineering in Medicine and Biology Society, (2007), pp.2315-2318.

[12] Cantoni V, Lombardi L \& Lombardi P, "Challenges for data mining in distributed sensor networks", IEEE 18th International Conference on Pattern Recognition, Vol.1, (2006), pp.1000-1007.

[13] Olfati-Saber R \& Murray RM, "Consensus problems in networks of agents with switching topology and time-delays", IEEE Transactions on automatic control, Vol.49, No.9, (2004), pp.1520-1533.

[14] Kiziroglou ME, Boyle DE, Yeatman EM \& Cilliers JJ, "Opportunities for sensing systems in mining", IEEE Transactions on Industrial Informatics, Vol.13, No.1, (2017), pp.278-286.

[15] Lian J, Naik K \& Agnew GB, "Data capacity improvement of wireless sensor networks using non-uniform sensor distribution", International Journal of Distributed Sensor Networks, Vol.2, No.2 (2006), pp.121-145.

[16] Huang CJ, Chuang YT \& $\mathrm{Hu} \mathrm{KW}$, "Using particle swam optimization for QoS in ad-hoc multicast", Engineering Applications of Artificial Intelligence, Vol.22, No.8, (2009), pp.1188-1193.

[17] Bari A, Wazed S, Jaekel A \& Bandyopadhyay S, "A genetic algorithm based approach for energy efficient routing in two-tiered sensor networks", Ad Hoc Networks, Vol.7, No.(4), (2009), pp.665676 .

[18] Araújo AFR \& Garrozi C, "MulRoGA: A multicast routing genetic algorithm approach considering multiple objectives", Applied Intelligence, Vol.32, (2010), pp.330-345.

[19] Lindsey S \& Raghavendra CS, "PEGASIS: Power-Efficien Gathering in Sensor Information Systems", SIGMOD Record, Vol.32, No.4, (2003), pp.66-71.

[20] Jeong JK, Kim SO \& Song CH, "A solution of search multicast routing tree based on extended simulated annealing algorithm", WSEAS Transactions on Mathematics, Vol.4, (2005), pp. 218-223. 